\title{
MCT A: Target Tracking Algorithm based on Minimal Contour in Wireless Sensor Networks
}

\author{
Jaehoon Jeong, Taehyun Hwang, Tian He, and David Du \\ Department of Computer Science \& Engineering \\ University of Minnesota \\ Minneapolis, MN 55455, USA \\ Email: \{jjeong,thwang,tianhe,du\}@cs.umn.edu
}

\begin{abstract}
This paper proposes a minimal contour tracking algorithm $(M C T A)$ that reduces energy consumption for tracking mobile targets in wireless sensor networks in terms of sensing and communication energy consumption. $M C T A$ conserves energy by letting only a minimum number of sensor nodes participate in communication and perform sensing for target tracking. $M C T A$ uses the minimal tracking area based on the vehicular kinematics. The modeling of target's kinematics allows for pruning out part of the tracking area that cannot be mechanically visited by the mobile target within scheduled time. So, $M C T A$ sends the tracking area information to only the sensor nodes within minimal tracking area and wakes them up. Compared to the legacy scheme which uses circle-based tracking area, our proposed scheme uses less number of sensors for tracking in both communication and sensing without target missing. Through simulation, we show that $M C T A$ outperforms the circle-based scheme with about $60 \%$ energy saving under certain ideal situations.
\end{abstract}

\section{INTRODUCTION}

The energy efficiency is one of the important research issues in wireless sensor networks since it determines the lifetime of the sensor network deployed for the intended applications, such as environmental monitoring, area surveillance, and target tracking. Especially, in the target tracking application, the energy efficiency is the most important factor as it leads to the long-lived target tracking. In the target tracking setting, an energy-aware target tracking algorithm not only should guarantee the tracking of mobile targets (e.g., enemy tanks or vehicles), but also should maximize the sensor network lifetime using a minimum number of working sensor nodes. The tracking area is defined as the possible region where the mobile target can reach from its current position during some limited time. The legacy tracking scheme [1], [2] uses the circle-based tracking area for simplicity. Since the mobile target, such as vehicle, moves according to its vehicular kinematics [3], it is impossible for it to reach all the area of the tracking circle. We found that we can reduce the number of working sensor nodes in each tracking area if we use the vehicular kinematics that the mobile target moves according to. We try to prune out from the tracking circle the most unlikely region that the target cannot visit during some limited time. This makes the tracking area be a minimal-sized area based on the vehicular kinematics. Only the sensors within the minimal tracking area work for target tracking during some limited time. Thus, by updating the minimal tracking area containing the mobile target during the target's trajectory, the sensor network based on our scheme consumes less energy than the legacy scheme based on tracking circle. We call our minimal tracking area the minimal contour.

Our contributions in this paper are as follows:

- The modeling of tracking area based on the vehicular kinematics.

- The optimization of tracking contour.

- The minimization of communication energy consumption.

- The considerations on measurement errors for mobile target's movement.

The rest of this paper is organized as follows: Section II describes the problem formulation for mobile target tracking. Section III explains the minimal contour tracking algorithm, the modeling of tracking contour, the optimization of tracking contour, the minimization of communication energy consumption, and the measurement error handling. In Section IV, we show that our contour scheme outperforms the legacy scheme based on tracking circle through simulation. We summarize our work and shed our future work in Section V.

\section{Problem Formulation}

We propose an energy-aware target tracking algorithm based on tracking contour in order to maximize the lifetime of the sensor network. The tracking contour is constructed based on the vehicular kinematics, which allows a minimal number of sensors near to the target to work in both communication and sensing.

\section{A. Assumptions and Definitions}

We have a few assumptions as follows:

- The sensing range is a uniform-disk whose radius is $r$.

- The communication radius is adjustable by controlling RF transmission power [11], [12].

- The RF transmission angle is adjustable by using directional antenna [13]-[15].

- The localization scheme is provided for the sensor nodes in order to find the position, speed, and direction of the vehicle at any time [4], [5].

We define four terms as follows:

Definition 1. Refresh Time. We define the lifetime of of the tracking area as refresh time. The old tracking area is replaced 
with the new tracking area according to the target's movement every refresh time.

Definition 2. Tracking Circle. The tracking circle is the tracking area where the target can visit for its current position and speed during refresh time. The tracking circle's radius is the multiplication of target's speed and refresh time.

Definition 3. Tracking Contour. The tracking contour is the tracking area where the target can visit for its current position, speed and direction during refresh time, considering the vehicular kinematics. It prunes out the most unlikely area from the tracking circle.

Definition 4. Minimal Contour. The minimal contour is a tracking contour for a given target's speed that allows for the minimization of energy cost spent for target tracking.

\section{B. Main Idea}

Our main idea is to minimize the tracking area used to determine the neighboring sensors that participate in target tracking. The legacy scheme always uses a tracking circle surrounding the mobile target that is modeled as a random walk. Though this approach is simple, more than a half of the tracking area based on circle cannot be visited by the target within some limited time [3]. Our scheme uses the vehicular kinematics to prune out the most unlikely area where the target cannot visit within such small time. Our tracking contour's shape changes from a circle to a contour (e.g., cone-like shape) according to the target's movement state (i.e., stopping state and moving state). Our model for tracking contour is represented as a polygon approximately including the area where the target can reach during refresh time based on the vehicular kinematics. Figure 1 shows two tracking areas: (a) Tracking Circle and (b) Tracking Contour. Let $p=(x, y)$ be the target's position vector where $x$ is $x$-coordinate and $y$ is $y$-coordinate. Let $m=(v, \theta)$ be the target's movement vector where $v$ is the target's speed and $\theta$ is its direction. We can see that the contour's area is always the subset of the circle's area. So, the contour can allow fewer sensor nodes to track the target than the circle; that is, only the sensor nodes within the contour whose area is smaller than the circle's perform sensing work, leading to energy saving.

Figure 2 shows the possible trajectories of the vehicle according to refresh time where a new contour is generated for tracking every refresh time. Let one turning time be the time that is needed for the vehicle whose speed is $v$ and whose turning angle is its maximum steering angle $\phi$. Figure 2(a), Figure 2(b), Figure 2(c), and Figure 2(d) show the tracking area for $\frac{1}{4}$ turning time, that of $\frac{2}{4}$ turning time, that of $\frac{3}{4}$ turning time, and that of one turning time, respectively. The outer circle in each figure indicates the tracking area predicted by the legacy scheme based on circle. Thus, the tracking area is determined with refresh time, vehicle speed, and turning angle. Thus, since only sensor nodes which belong to the tracking contour smaller than the tracking circle need to turn on their sensing and communication devices, our scheme based on tracking contour can save more energy than the legacy scheme based on circle.

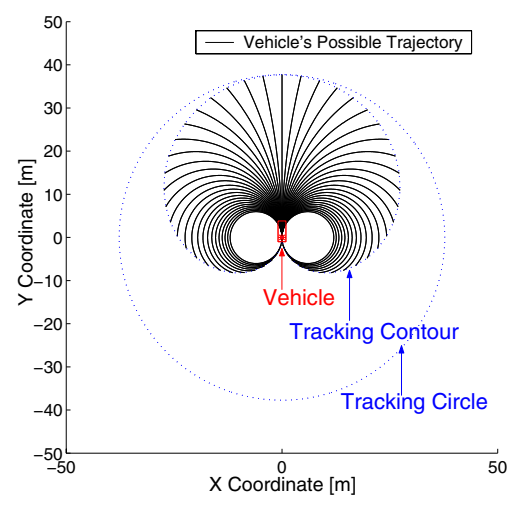

Fig. 1. Tracking Area: Tracking Contour versus Tracking Circle

\section{Design Goals}

We have three design goals to minimize the energy consumption for target tracking: (a) the optimization of refresh time for minimal contour, (b) the minimization of communication cost in terms of the number of RF receiving sensors, and (c) each sensor's localized determination of its warmingup time and finishing time for sensing.

The refresh time determines the size of contour given the target's speed; that is, the bigger the refresh time is, the bigger the contour is. We need to use the optimal refresh time that leads to the minimal energy consumption for target tracking. This refresh time is selected as an optimal time, considering all the energy costs for tracking, such as communication cost, computation cost, and sensing cost.

The $R F$ transmission power control and directional antenna technology are adapted for reducing the communication cost. Because the receiving power consumption is dominant factor in energy cost, we should reduce it. The RF transmission power control and directional antenna technology allow to save receiving power consumption.

When the sensor nodes turn on and turn off their sensing devices can be decided locally in order to save their energy with the target's movement information (i.e., position and speed), refresh time and their own position.

With the given number of sensor nodes, our objective is to maximize the sensor network lifetime to satisfy the following conditions:

- to guarantee the target tracking without missing and

- to use the minimal contour appropriate for the target's speed in terms of the energy cost in both communication and sensing.

\section{Minimal Contour Tracking Algorithm (MCTA)}

Assume that the sensor detecting the target can know the position and speed of the mobile target through the target localization scheme [4], [5]. We define a sensor node disseminating the tracking contour information as root node. When a sensor plays a role of root node, it broadcasts the movement information of a mobile target.

When the sensor node receives the broadcasted message containing the minimal contour information, it determines 


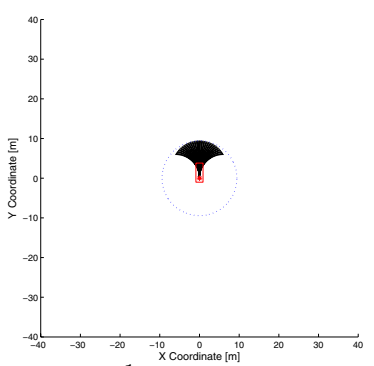

(a) $\frac{1}{4}$ Turning Time

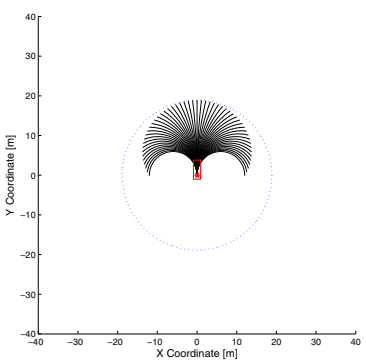

(b) $\frac{2}{4}$ Turning Time

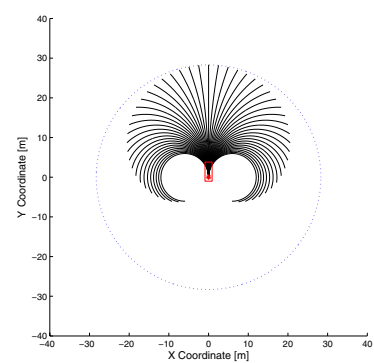

(c) $\frac{3}{4}$ Turning Time

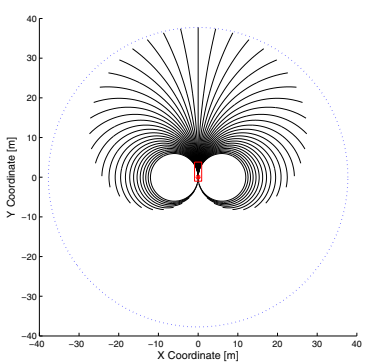

(d) One Turning Time

Fig. 2. Tracking Contour's Shape according to Refresh Time

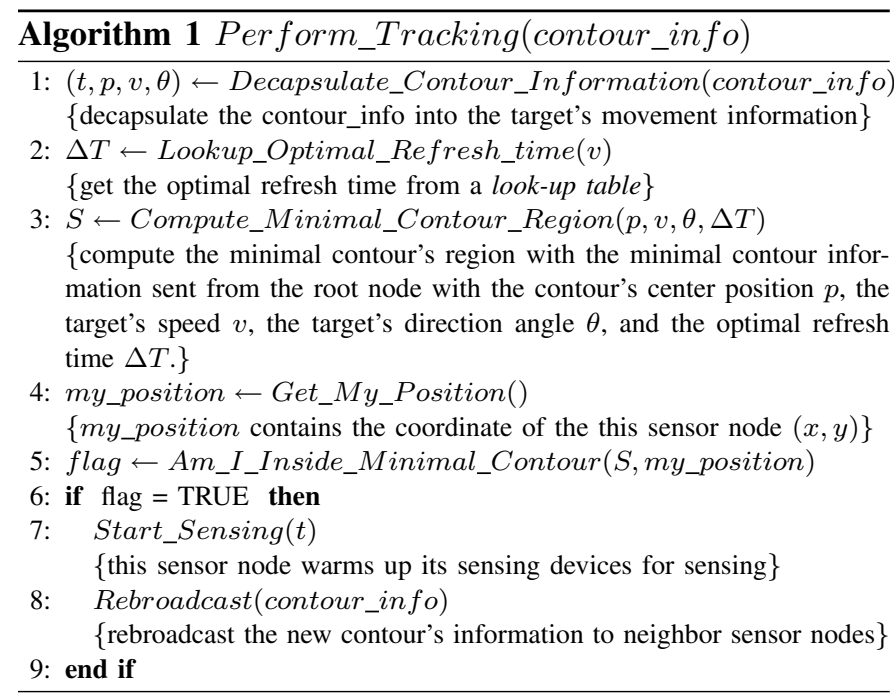

whether it belongs to the minimal contour or not. If the sensor is the member of the new contour, it warms up its sensing devices to prepare for the target tracking and relays the message to its neighbor sensor nodes. Otherwise, it just relays the message to its neighbors.

\section{A. Modeling of Vehicle Motion}

We assume that the mobile target is a four-wheeled vehicle. We can define the vehicle motion based on the vehicular kinematics [3], [9]. Refer to our technical report for the detailed discussion [18].

\section{B. Modeling of Tracking Contour}

We can make a tracking contour using the vehicular kinematics discussed in Section III-A. Let $\left(X_{0}, Y_{0}\right)$ be the target's current position, $\Theta_{0}$ be the target's direction, and $V_{0}$ be target's speed. Let $\Delta T$ be refresh time. Let $\left(X_{\Delta T}, Y_{\Delta T}\right)$ be the target's position after $\Delta T$. We divide target movement into three kinds: (a) Straight movement, (b) Left turning, and (c) Right turning. We can make a polygon representing the tracking contour with the three styles of movement. Figure 3 shows the procedure constructing the tracking contour. The straight movement gives two points in like Figure 3(a). The first point is the target's current position $\left(X_{0}, Y_{0}\right)$. The second $\left(X_{\Delta T}, Y_{\Delta T}\right)$ is the point away from $\left(X_{0}, Y_{0}\right)$ by the distance that the target can go with its current speed and maximum acceleration. The point $\left(X_{\Delta T}, Y_{\Delta T}\right)$ can be computed through the vehicle motion process [18]. Like in Figure 3(b), the left points can be obtained through the vehicle motion process [18] by changing the steering angle from 0 to maximum steering angle discretely to the left. In the same way, the right points can be obtained by changing the steering angle from 0 to maximum steering angle discretely to the right. The obtained points construct a polygon like Figure 3(d). This polygon is used by each sensor to determine whether it should work for tracking. Only the sensors inside the polygon work, and other sensors continue to be idle. The inside checking is done by Ray Crossings algorithm [7].

When the refresh time is less than one turning time of the target, the tracking contour guarantees the tracking of the moving target without missing. Since the tracking contour covers all the possible area visited by the mobile target, it guarantees the no-missing tracking. But when the refresh time is bigger than one turning time, it is very hard to represent a tracking contour less than the tracking circle. In fact, in most cases, as the optimal refresh time is less than one turning time through the optimization of refresh time, we need not worry about the case where the refresh time is bigger than one turning time. Besides, the fast moving target cannot make a sharp turn to the left or to the right with its maximum steering angle since the maximum turning makes the target be overturned.

\section{Optimization of Refresh Time for Minimal Contour}

We need to use an optimal refresh time to let the contourbased tracking consume the minimum energy for target tracking. We optimize the refresh time according to the target's current speed. Refer to our technical report for the detailed discussion [18].

\section{Minimization of Communication Cost}

Since the communication cost is dominant factor in energy consumption in the target tracking, it is worthy to find out how to reduce such cost in our contour solution. The key idea is to reduce the communication energy cost using both RF communication range adjustment and directional antenna along with the minimal contour. Refer to our technical report for the detailed discussion [18]. 


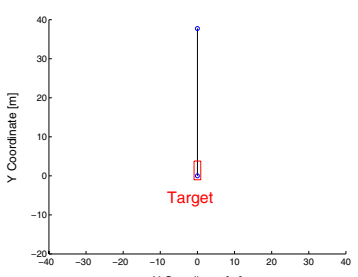

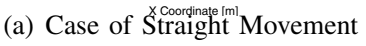

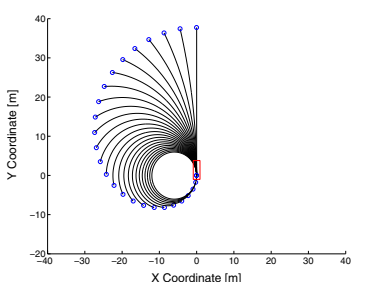

(b) Case of Left Turning

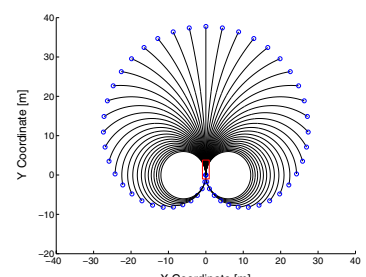

(c) Case of Right Turning

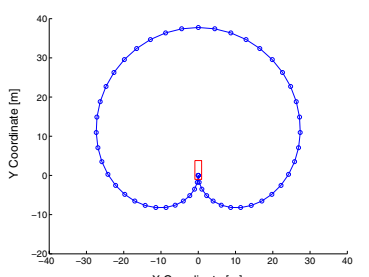

(d) Completed Tracking Contour

Fig. 3. Construction Procedure of Tracking Contour

\section{E. Handling of Measurement Errors for Target Localization}

The target localization is used to locate the most likely position of the mobile target with several sensor nodes that detected the mobile target at the same time [4], [5]. In order to estimate the mobile target's direction and speed, more than two localizations are needed where each localization provides a pair of the time and target position. We have assumed so far that the localization is performed to give these target's current position, speed and direction. However, in reality, since there are measurement errors in every localization scheme, we need to consider them to make a more realistic tracking contour. Refer to our technical report for the detailed discussion [18].

\section{Performance Evaluation}

We model the sensor network including sensor and vehicle on the basis of SMPL simulation model along with Matlab where SMPL is one of the discrete event driven simulators [8], [16].

\section{A. Simulation Analysis}

We define the sensor network lifetime as the time until at least one sensor node among the sensor nodes on the surveillance field dies due to the energy exhaustion.

The simulation environment is as follows:

- 10,000 sensor nodes are uniformly deployed in the surveillance field of $500[\mathrm{~m}] \times 500[\mathrm{~m}]$.

- The radius of communication is $75[\mathrm{~m}]$.

- The vehicle's speed is $30[\mathrm{~km} / \mathrm{h}]$ and its maximum turning angle is $25^{\circ}$.

We simulated to know the number of sensing sensor nodes, the number of receiving sensor nodes, and the cumulative energy consumption according to the vehicle's movement with the tracking circle and tracking contour, respectively. Figure 4 shows these three kinds of performance comparison. Let $X_{t}$ be the number of working sensor nodes at time $t$ for the contour based scheme and $Y_{t}$ be that at time $t$ for the circle based scheme. Let $E\left[X_{t}\right]$ be the average number of working sensor nodes for the contour based scheme and $E\left[Y_{t}\right]$ be that for for the circle based scheme. Let $\operatorname{Var}\left[X_{t}\right]$ and $\operatorname{Var}\left[Y_{t}\right]$ be the variances of $X_{t}$ and $Y_{t}$, respectively. Table I shows the comparison between two tracking schemes in terms of of the number of sensing sensor nodes in our simulation scenario. We can see that the ratio of the expected number of working (i.e., sensing) sensor nodes in contour based scheme to that
TABLE I

Measurement of Number of SENSING SENSORS

\begin{tabular}{|c|c|c|c|}
\hline Metric & Contour $\left(X_{t}\right)$ & Circle $\left(Y_{t}\right)$ & Ratio \\
\hline Area & $24.1\left[\mathrm{~m}^{2}\right]$ & $137.8\left[\mathrm{~m}^{2}\right]$ & 0.18 \\
\hline Expectation $(E)$ & 17 & 44 & 0.39 \\
\hline Variance $($ Var $)$ & 35.6 & 54.5 & 0.65 \\
\hline
\end{tabular}

in circle based scheme is about 0.25 time, equal to the area ratio $(0.18)$ where the contour's area is $24.14\left[\mathrm{~m}^{2}\right]$ and circle's area is $137.78\left[\mathrm{~m}^{2}\right]$. Therefore, we can conclude that we can reduce the number of working sensor nodes with our minimal contour scheme, maximizing the sensor network lifetime. Note that in Figure 4(a), the number of sensing sensors at the first refresh time is the same in two schemes. The reason is that the tracking contour cannot have enough information for the vehicle's movement at first, so should use the tracking circle.

The cumulative energy consumptions for two schemes are shown in Table II. When we do not use both RF transmission power control and directional antenna, the performance ratio of two tracking schemes is only 0.71 ; that is, the tracking contour can improve only $29 \%$ of the tracking circle's performance. The reason is that though $25 \%$ of sensors in tracking circle are used in tracking contour, the communication cost that is the major factor in energy cost is the same in two schemes [18]. To improve the performance in terms of energy cost, we need to use the RF transmission power control and directional antenna discussed in Section III-D. Like in Figure 4(b), we can reduce a large number of receiving sensor nodes with both tracking contour and two communication technologies. The tracking contour with two technologies can improve about $61 \%$ of the tracking circle that also uses the RF transmission power control. Note that it is good to use the tracking contour only with the RF transmission power control and without the directional antenna since it still brings the improvement of $56 \%$.

\section{CONCLUSION}

In this paper, we suggested a target tracking algorithm $M C T A$ using minimal tracking area called tracking contour that is based on the vehicular kinematics. MCTA minimizes the number of working sensor nodes in terms of the communication and sensing energy cost during the mobile target's trajectory. We showed that the ratio of tracking contour's working sensor number to tracking circle's working sensor number 


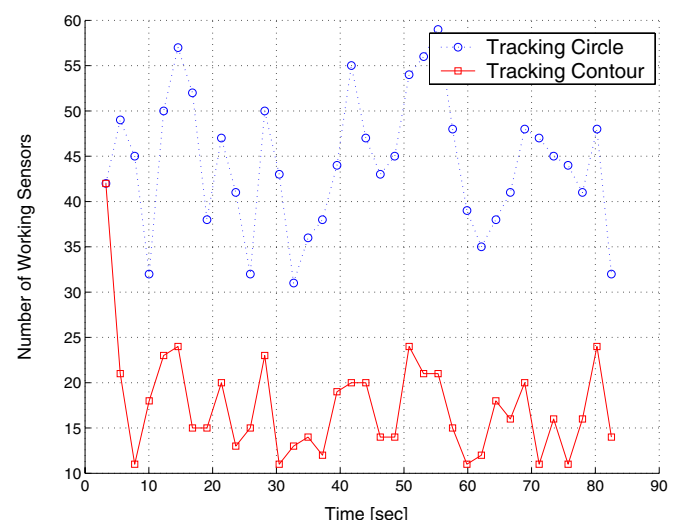

(a) Number of Sensing Sensor Nodes Every Refresh Time

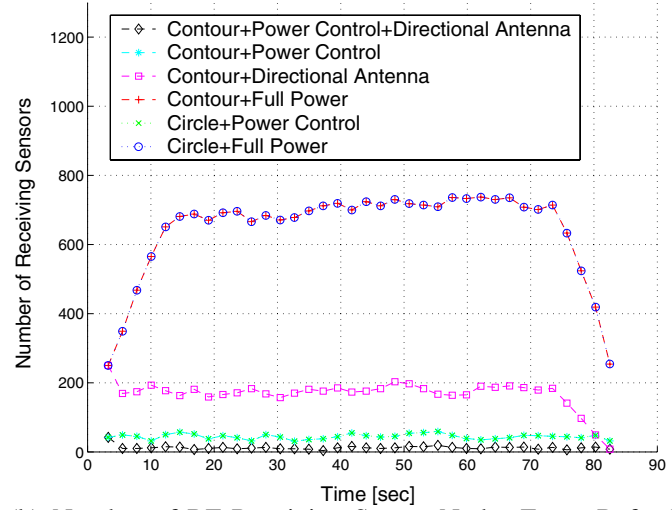

(b) Number of RF Receiving Sensor Nodes Every Refresh Time

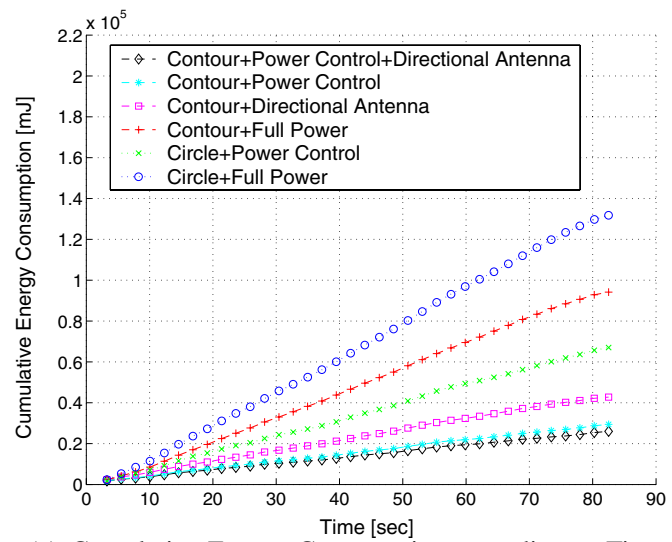

(c) Cumulative Energy Consumption according to Time

Fig. 4. Performance Comparison between Tracking Circle and Tracking Contour

TABLE II

COMPARISON OF CUMULATIVE ENERGY CONSUMPTION

\begin{tabular}{|c|c|c|}
\hline Method & Contour & Circle \\
\hline $\begin{array}{c}\text { Maximum Transmission Range } \\
\text { (i.e., Full Power) }\end{array}$ & $94166[\mathrm{~mJ}]$ & $131770[\mathrm{~mJ}]$ \\
\hline Directional Antenna & $42800[\mathrm{~mJ}]$ & N/A \\
\hline RF Tx Power Control & $29438[\mathrm{~mJ}]$ & $67045[\mathrm{~mJ}]$ \\
\hline $\begin{array}{c}\text { RF Tx Power Control and } \\
\text { Directional Antenna }\end{array}$ & $25988[\mathrm{~mJ}]$ & N/A \\
\hline
\end{tabular}

is proportional to the ratio of the tracking contour's area to tracking circle's area. This indicates that the reduction of the tracking area leads to the communication and sensing energy saving. We optimize the refresh time for minimal contour according to the vehicle current speed. Also, in order to reduce the dissemination of tracking contour information within the tracking contour, we used the $R F$ transmission power control and directional antenna, leading to the minimization of the number of RF receiving sensors. As our future work, we will implement our tracking algorithm in real sensor nodes (e.g., Mica [10]) and test it in our indoor testbed.

\section{ACKNOWLEDGMENT}

This work was supported by the Department of Computer Science and Engineering and Digital Technology Center at the University of Minnesota.

\section{REFERENCES}

[1] W. Zhang and G. Cao, Dynamic Convoy Tree-Based Collaboration for Target Tracking in Sensor Networks, IEEE Transactions on Wireless Communications, Vol. 3, No. 5, September 2004.

[2] W. Zhang and G. Cao, Optimizing Tree Reconfiguration for Mobile Target Tracking in Sensor Networks, IEEE Infocom, March 2004.

[3] S. M. LaValle, Planning Algorithms, Cambridge University Press, 2006.

[4] T. He, C. Huang, B. M. Blum, J. A. Stankovic and T. Abdelzaher, Range-Free Localization Schemes for Large Scale Sensor Networks, ACM Mobicom, September 2003.

[5] J. Lee, K. Cho, S. Lee, T. Kwon and Y. Choi, Distributed and energyefficient target localization and tracking in wireless sensor networks, Elsevier Computer Communications (COMCOM), Vol. 29, No. 13, pp. 2494-2505, August 2006.

[6] D. R. Kincaid and W. W. Cheney, Numerical Analysis: the Mathematics of Scientific Computing, Van Nostrand, 1991.

[7] J. O'Rourke, Computational Geometry in $C, 2^{\text {nd }}$ Edition, Cambridge University Press, 1998.

[8] M. H. MacDougall, Simulating Computer Systems: Techniques and Tools, MIT Press, 1987.

[9] S. J. Maybank, A. D. Worrall and G. D. Sullivan, Filter for Car Tracking Based on Acceleration and Steering Angle, British Machine Vision Conference, 1996.

[10] J. L. Hill and D. E. Culler, Mica: a Wireless Platform for Deeply Embedded Networks, IEEE Micro, Vol. 22, Nov/Dec 2002.

[11] V. and P. R. Kumar, Principles and Protocols for Power Control in Wireless Ad Hoc Networks, IEEE J. Sel. Areas Commun. (JSAC), Vol. 1, pp.76-88, January 2005.

[12] V. Kawadia and P. R. Kumar, Power Control and Clustering in Ad Hoc Networks, IEEE Infocom, March 2003.

[13] Y. Ko, V. Shankarkumar and N. H. Vaidya, Medium Access Control Protocols Using Directional Antennas in Ad Hoc Networks, IEEE Infocom, March 1999.

[14] Q. Tian, S. Bandyopadhyay and E. J. Coyle, Effect of Directional Antennas on Spatiotemporal Sampling in Clustered Sensor Networks, IEEE WCNC, April 2006.

[15] Sensor Network Project for Directional Antenna, https://engineering.purdue.edu/IDEAS/SensorNtwks.html

[16] Matlab of the MathWorks, http://www.mathworks.com

[17] A. Aljadhai and T. F. Znati, Predictive Mobility Support for QoS Provisioning in Mobile Wireless Environments, IEEE Journal on Selected Areas in Communications (JSAC), Vol. 19, No. 10, October 2001.

[18] J. Jeong, T. Hwang, T. He and D. Du, MCT A: Target Tracking Algorithm based on Minimal Contour in Wireless Sensor Networks, Technical Report of University of Minnesota, No. 07-002, January 2007: http:// www.cs.umn.edu/research/technical_reports.php?page=year\&year=2007 